\title{
Multicultural Competencies of Healthcare Professionals and Disability-Inclusive in Telehealth during the COVID-19 Pandemic
}

\author{
Si-Yi Chao ${ }^{1 *}$, Keith B Wilson ${ }^{1}$ and Carrie L Acklin ${ }^{2}$ \\ ${ }^{1}$ Department of Early Childhood, Special Education, and Counselor Education, \\ University of Kentucky, USA \\ ${ }^{2}$ Department of Rehabilitation and Counseling, University of Wisconsin-Stout, USA \\ *Corresponding Author: Si-Yi Chao, Department of Early Childhood, Special \\ Education, and Counselor Education, University of Kentucky, Lexington, KY, USA.
}

Received: September 30, 2020

Published: November 30, 2020

(C) All rights are reserved by Si-Yi Chao., et al.

\section{Abstract}

There is evidence that people with disabilities are more likely to be discriminated against and to have disproportionate health care disparities compared to people who are able-bodied during the devastating COVID-19 pandemic. In light of COVID-19, telehealth has been transformed to the primary health care delivery due to social restrictions of most, globally. Disability-inclusive telehealth design can promote culturally diverse groups access and more accurate COVID-19 information, knowledge, public health measures, and guidelines of preventive strategies for acquiring COVID-19. Applying multicultural competencies is required for healthcare providers to maximize health engagement and outcomes of PWD. In many areas of health care, healthcare professionals recognize needs, expectations, and perceptions of diverse disability populations. That being said, healthcare professionals should implement appropriate interactions with a patient with a disability and provide culturally responsive telehealth services for other underrepresented groups as well. Hence, the multicultural competencies of healthcare professionals and disability responsive telehealth systems can assist PWD to mitigate health care disparities.

Keywords: People with Disabilities; COVID-19; Multicultural Competencies; Disability Inclusive; Telehealth; Accommodations

\section{Abbreviations}

COVID-19: The Novel Coronavirus Disease; WHO: World Health Organization; CDC: Centers for Disease Control and Prevention; US: United States; PWD: People with Disabilities; PPE: Personal Protective Equipment; LGBTQIA+: Lesbian, Gay, Bisexual, Transgender, Queer/Questioning, Intersex, Asexual/Agender and Other Sexual Orientations; SES: Socioeconomic Status; SCI: Spinal Cord Injury; LGBT: Lesbian, Gay, Bisexual, and Transgender; HHS: Health and Human Services; HIPAA: Health Insurance Portability and Accountability Act; HRSA: Health Resources and Services Administration; ADHD: Attention Deficit Hyperactivity Disorder

\section{Introduction}

The Novel Coronavirus Disease (COVID-19) pandemic is destroying individuals, communities, societies, and countries all over the world. In addition, there are other areas that have been impacted by COVID-19, such as health care, education, employment, economic, sociocultural and political aspects around the world. To provide a disability perspective, over 1 billion people globally live with a certain type of disability and people with disabilities (PWD) represent $15 \%$ of the world population [1]. According to the World Health Organization (WHO) and the Centers for Disease Control and Prevention (CDC) in the United States (US), PWD is one of the most vulnerable populations that are more likely to be infected COVID-19. Thus, PWD are likely to have higher mortality rates by the Coronavirus. The WHO published a guideline and action plan about considering PWD during COVID-19 outbreak. These guidelines consisted of health care accessibility, building up support systems, physical and mental health considerations of individuals with disabilities, families and caregivers, suggested crisis responses and 
actions of the government, human service providers, resident institutions and communities for protecting PWDs' health and life [2]. As a consequence of the COVID-19 pandemic, telehealth has been adopted rapidly to replace traditional in-person health care and home visit care because of social distance restrictions regulated by the CDC and others [3]. The utilization of telehealth services is more likely to become a norm even after the COVID-19 pandemic. Before COVID-19 outbreak, PWD perceived health care disparities focusing on in-person visit attributing to: (a) the lack of awareness of health service provider (e.g. doctors, nurses, therapists, mental health counselors) about disability culture diversity between different disability types, (b) difficulty of understanding information and healthcare service communication, and (c) inaccessibility of physical building, tests, and examination equipment [3]. Recently, telehealth needs and barriers of PWD should be re-examined due to social restrictions made by COVID-19 outbreak. Redesigning the continuum of care in an equitable and culturally responsive way in telehealth would generate positive care outcomes and diminish challenges for underrepresented populations. In addition, training multicultural competent healthcare professionals during digital care delivery beyond COVID-19 is becoming fundamental for telehealth equity and quality. To provide quality care to PWD and other underrepresented groups, healthcare professionals and public health experts should dedicate multicultural considerations and efforts in telehealth access and quality to reduce health care disparities and social inequalities.

In this conceptual article, the first section is focused on discussing health care inequalities and unprotected rights that PWD have encountered during the COVID-19 pandemic. Second, how existing health care disparities and discrimination are perceived by PWD. Third, what are telehealth benefits and possible challenges for PWD in health care delivery during the COVID-19 pandemic. The final section of this manuscript illustrates how to apply multicultural competencies for healthcare professionals and to construct disability-inclusive telehealth technology for PWD. Finally, this study would like to raise stakeholders' (e.g. regulators, governments, healthcare providers, telehealth vendors, disability advocacy activists, educators) awareness and actions about health care access rights and disparities of people with disabilities resulting from COVID-19. The importance of multicultural competencies of healthcare providers, telehealth inclusion and accommodation, and disability-inclusive regulations by policymakers and the government in telehealth and health care delivery are emphasized and advocated for the vulnerable disability community.
People with disabilities during COVID-19

In the US, approximately $12.6 \%$ of individuals reported having at least one disability [4]. However, PWD are continuing to encounter the lack of accessibility to health care and human services, poverty, a deficit of insurance coverage, low education attainment, high unemployment rate, aging, microaggression, and social oppression compared to people with able-bodies [1]. Certainly, PWD is the largest underrepresented and vulnerable community in the US. This disability community has also lived with negative social attitudes and structural inequity projected upon them, which hinders their access to healthcare services. Thus, PWD and their quality of life is hampered even though they have needs that are increasingly evaluated $[5,6]$.

While the US federal and state governments respond to the COVID-19 pandemic, PWD's needs and public health measures have not been considered for appropriate crisis plans to eliminate potential infectious risks for disability groups [7-9]. Until recently, no specific data set, data analysis, physical and mental health and function reports, quality of life metrics, test access information, and mortality and morbidity rates are presented related to the disability population in the US public health system and the CDC database [10]. The current situations and barriers PWD have encountered are still unclear to address the level of disparities to establish national crisis response action plans for PWD in the US [11]. Additionally, due to mortality and morbidity data discrepancy of PWD and abled-bodied people, human service institutions and policymakers have difficulties in being aware disparities that PWD have continuously faced in health care, education, housing transportation, and employment aspects. PWD have concerned with receiving less lifesaving treatments and disproportionately influenced by public health measures implemented by the governments to control the virus spread [7].

The United Nations and the WHO have released several guidelines and articles about disability-inclusive responses during COVID-19 [7,12]. Simultaneously, PWD living in the US are facing: (a) inaccessibility of public health information, testing, and treatments, (b) health care and human service disparities, (c) food, housing, employment, financial, and life insecurity, (d) loss of social support, (e) lack of personal protective equipment (PPE), including medical masks, hand sanitizers, and hygiene materials and (f) no transportation accessibility during the COVID-19 pandemic. Impacted by COVID-19 outbreak, PWD, who cannot follow social 
distancing, are more likely to expose themselves to the risks of contracting the virus, especially if they do not have PPE availability. Additionally, PWD might have higher morbidity rates because of COVID-19. Many of them live with other chronic disorders and underlying health conditions (e.g. diabetes, respiratory disorders, heart diseases, chronic renal diseases). During COVID-19 outbreak, some people with severe cardiopulmonary and respiratory issues felt fear, stress and deprioritization to be rejected and deprived of their essential treatments and ventilator equipment [13]. Several disability advocacy groups have urged the Department of Health and Human Services to examine the appropriateness of the state's health care rationing plans for PWD about basic rights assurance of receiving needed health care. Unsecured health and safety have exaggerated an individual with a disability's physical, psychological, and emotional distress.

Health care disparities in the diverse disability community

It is reported that PWD have disproportionate health disparities and a lower quality of life when compared to their able-bodied counterparts. For example, Moscoso-Porras., et al. found that PWD are less willing to seek health care because of perceived discrimination against their disabilities [14]. Furthermore, people with communication disabilities and physical disabilities were more likely to perceive discrimination than people who may be abled-bodied. The aforementioned results are supported by other findings that underrepresented groups [e.g. racial and ethnic minorities, religion, lesbian, gay, bisexual, transgender, queer/questioning, intersex, asexual/agender and other sexual orientations (LGBTQIA+) community, low socioeconomic status (SES), immigrants and refugees) are less likely to seek and utilize health services whether they perceived discrimination in healthcare facilities, communities, and the society in which they live. To this point, in Iezzoni's qualitative study, thirty-five interviewees with different disability types (e.g. traumatic brain injury, cerebral palsy, arthritis, heart conditions, spinal cord injury, hearing low, blindness and low vision) reported several barriers that hindered their primary health care quality in rural America [15]. These barriers included difficulties of: (a) finding caring and competent physicians, (b) continuing need for specialists and sophisticated services, (c) poverty and heavy reliance on Medicaid, (d) physical access to healthcare settings, (e) barriers to local and long-distance travel, and (f) need for advocacy and information resources. According to PWD's shared experiences in the rural area in Iezzoni's study, PWD perceived less disability responsive healthcare services provided in the rural area compared to those in urban area [15]. Intersecting disability and other characteristics and identities (e.g. geographic, socioeconomic status) of a person with a disability would perceive double even triple discrimination and marginalization in health care access.

Let us look at another study that focused on patients with intellectual disabilities. Ali., et al. interviewed twelve patients with intellectual disabilities and twelve caregivers about their perceived discrimination while interacting with physicians and other healthcare providers [16]. The results indicated that the patients with intellectual disabilities felt ignored during medical consultations by clinical practitioners who usually talked to their attendants instead of communicating with them. Also, healthcare providers failed to make accommodations to their communication for patients with intellectual disabilities. For instance, clinical practitioners were talking too fast, not providing sufficient response time for patients with intellectual disabilities to respond, providing too much information at one time, and not using understandable language for information exchange and explanations. Some of the patients with intellectual disabilities reported that healthcare providers treated them as a child, not voluntarily explaining any diagnosis, progress, medical procedures, and treatment plans. The clinicians and staff might assume their patients with intellectual disabilities do not understand and feel challenges of communicating to them. The caregivers also felt that the clinicians had no awareness to empathize with patients with intellectual disabilities who cannot express their health conditions and concerns immediately and explicitly. If the clinicians gave more time and asked more specific questions using simple language, patients with intellectual disabilities can exchange detailed information for further diagnosis and treatment plans. Perceiving discriminations during health care delivery, patients with intellectual disabilities losing their trust of healthcare professionals, prohibited their willingness to access and utilize health care. Professional multicultural competencies of healthcare providers are necessary to establish rapport with PWD and increase positive health outcomes.

The cultural competence of clinical practitioners was associated with satisfaction and quality of care of clients [17]. Michalopoulou., et al. illustrated that $44.7 \%$ of respondents with undecremented races and disabilities strongly agreed that they felt satisfied with health care and functional outcomes attribute to clinical practitioners with better cultural skills [18]. Moreover, the satisfaction and functional outcomes were related to the process of care, including trust, communication, and respect from clinical practitioners. Pro- 
fessionals with appropriate and proficient multicultural competencies who apply knowledge and skills for clients during the service process can bring satisfaction to health and functional outcomes.

Likewise, multicultural competence training is critical for healthcare providers to provide appropriate and patient-centered services for PWD with diverse backgrounds. Burch designed a diverse training program for health care providers to examine the changes of their attitudes, knowledge and self-efficacy while treating people with spinal cord injury (SCI) who belong to LGBT community [19]. Approximately 400 healthcare providers participated a training program, including a pre-briefing questionnaire, a videotape, a post-briefing questionnaire and discussion sessions. The results from the pre-briefing questionnaire showed that $79 \%$ of the participants had never thought about diversity issues of patient with SCI who have diverse sexual orientations. These participants presented the lack of awareness of multicultural diversity of patients with SCI and expressed no willingness to change their behaviors. Approximately $71 \%$ of healthcare professionals reported their tolerance while serving patients with SCI who are LGBT, $19.6 \%$ of them showed some respect, and $7.8 \%$ of them had full respect toward people with SCI who belong to LGBT community. Also, $68 \%$ of healthcare providers reported very low to average level of multicultural knowledge. Approximately $70 \%$ of participants self-reported $0 \%$ to $20 \%$ confidence of providing culturally responsive healthcare services to patients with SCI who have diverse sexual orientations. Nevertheless, $64 \%$ of healthcare providers moderately agreed the positive effects on respect attitudes and the improvement of multicultural knowledge after a brief diversity training. Moreover, $80 \%$ of participants agreed that watching the videotape enhanced their confidence of culturally responsive service delivery for people with disabilities who belong to LGBT community. Therefore, appropriate multicultural competence training can foster healthcare professionals' attitudes, knowledge, and confidence in interacting with PWD from diverse cultural backgrounds and provide equitable health care.

Telehealth benefits and challenges for PWD during COVID-19

Telehealth as defined by the US Health Resources Services Administration (HRSA) is the use of electronic information and remote communication technologies between patients/clients and healthcare providers for personal healthcare services. Telehealth, including telemedicine, e-health, mobile health, connected health, telepsychiatry, telemental and telebahavioral health, and telecounseling, refers to a broader scope of remote health services
[20]. Telehealth services consist of: (a) remote clinical medical services covered under telemedicine (e.g. primary care, diagnosis, assessment, surgery consultation, medication consultation, routine health care), (b) remote mental and behavioral care services (e.g., mental health counseling, psychological therapies, substance abuse counseling), and (c) remote non-clinical services (e.g., public health education and promotion, nutrition education, wellness checking, following up, electronic health reports, and health administration). Patients/clients can utilize (a) asynchronous technologies, like electronic health report system, question box, e-mail, text, mobile applications, streaming media, store-and-forward imaging, and (b) synchronous ways, like video conferencing platforms and other telecommunication equipment for the delivery of health care interventions [21,22]. Telehealth has been developed with a variety of features and functions for healthcare providers easy to use.

Until recently, part of people with intellectual disabilities, developmental disabilities, and cognitive disabilities have less knowledge about what are the etiology and consequences if COVID-19 infects them? Why should they take COVID-19 outbreak seriously? Why the protective strategies, including mask-wearing, handwashing, and social distance, should be implemented and for what purpose. What the daily updated report and data delivered by the governor mean? The languages and visual images (e.g. graphs, pictures, animations) of communications on the news, social media, educative videos, and broadcasts are not plain and easy to understand for PWD who have specific needs. As a result, people with intellectual, developmental disabilities and cognitive disabilities find it hard to raise awareness, obtain knowledge, and implement protective steps in their daily lives. Incorporating culturally responsive and disability-inclusive into telehealth platform design promotes health care engagement of diverse disability community.

\section{Telehealth benefits for people with disabilities}

Due to COVID-19, telehealth plays an essential role in service delivery to decrease health care barriers resulting from stay-athome orders and social distance policies. Reporting on telehealth advantages, Agha., et al. believes that the advantages for PWD consisted of (a) time and cost effectiveness, including care and transportation, (b) supporting mutual information exchange between patients and healthcare professionals, (c) improving accessibility of primary and secondary care professionals for interdisciplinary communications, (d) decreasing need of personal care assistance, (e) increasing effectiveness of medication and prescription communication, and (f) decrease infectious risk with less exposure of 
people in public, especially during the COVID-19 pandemic [3,23]. For a person with a severe chronic disorder or complicated disorders, telehealth as a proper service could reduce contagious risks while traveling to in-person health care visits. For a person with a disability living in a rural area, telehealth can solve a shortage of experienced healthcare providers in interdisciplinary collaboration and knowledge sharing [24,25]. To that point, Brigo., et al. asserted that telehealth provides equivalent care in health management, symptom control, medication adherence, and emergency visits compared to traditional in-person visits [3]. As shown, Accessible, affordable, and inclusive telehealth for PWD can enhance their quality of life and wellbeing of PWD.

\section{Telehealth challenges for people with disabilities}

However, Annaswamy., et al. presented several challenges for telehealth providing for PWD [26]. The challenges include: (a) infrastructure and access in the local region, (b) operational and systems of telehealth platform, (c) telehealth regulatory inconsistency across local, state, insurance and federal levels, (d) communication between patients with disabilities and healthcare providers, including transparency of health education information delivery and lack of technology accommodation, and (e) multicultural competencies of healthcare providers and culturally responsive interactions with patients with disabilities. For instance, seldom technology accommodations, such as closed captioning, live captioning, or interpreting requests, are provided for deaf people during video conferences and phone calls with clinical practitioners. This lack of inclusion and disability responsive telehealth services exaggerate gaps of health care disparities, trust in healthcare providers, and health care utilization in the disability community.

The US Department of Health and Human Services (HHS) has taken actions to increase the flexibility of certain legislations under the Health Insurance Portability and Accountability Act of 1996 (HIPAA) to ensure the privacy and security of health information for all. The HRSA has funded and supported regional hospitals, primary care institutions, and community-based institutions which would like to provide telehealth during COVID-19. Policymaking also included reimbursement for healthcare providers' service delivery by different ways of telehealth, such as phone calls, text, emails, and video conferencing. The usage of telehealth services is demand-increasing for PWD that health care equity should be carefully considered in developing policies. Telehealthcare disparities in access, quality, effectiveness, and service experiences of PWD cannot be overlooked. Therefore, stakeholders, including lawmakers, telehealth developers, healthcare providers, and insurance payers, should ensure the expansion of telehealth in the advancement of care equity, including underrepresented patients who urgently need long-term telehealthcare plans. Meanwhile, healthcare professionals need to acknowledge their personal culture and worldviews when working with diverse disability communities and to reflect on self-biases that might produce discrimination and devalue perceived by PWD during service provision.

Lack of evidence-based practices of multicultural competencies in telehealth for PWD

In fact, telehealth has been determined its potential market and capability of facilitating health care delivery to PWD living in rural areas or difficult to travel [24]. Also, adequate trainings of cultural competencies and culturally inclusive health care can benefit outcomes of care, wellness, and quality of life for PWD [18,19,27]. Instead, it is unclear how implementation and quality of inclusive telehealthcare services are delivered by multicultural competent healthcare professionals to diverse PWD. Hilty., et al. systematically reviewed evidence-based studies about culturally competent telebehavioral health care for diverse patients with behavioral health disorders in rural areas [28]. Four key terms were focused on in this literature review, "cultural", "competencies", "telehealth" and "health." Merely, seven of 1,118 studies were associated with cultural, competencies, or telehealth. Few studies illustrated skills and competencies in telehealth combining with cultural diversity and inclusion for people with behavior health disorders. Most of behavioral health service providers received multicultural competence training and utilized interpreters and language service for diverse patients with behavioral health disorders. Whereas, no specific multicultural competence trainings integrating in telehealth or telebehavioral delivery were provided for behavioral health providers' professional development [28].

Moreover, Butler., et al. found that "cultural competence", "culturally responsive" or "multicultural competencies" were difficult to be identified in their systematically review of literature about culturally appropriate health care for children and adults with disabilities [29]. In addition, the terms as "remote medicine", "virtual care" or "telehealth" were included in disability literature searching. Butler., et al. considered virtual care as an intervention extending from culturally competent to decrease health disparities and increase healthcare access for PWD living in the rural area or 
having difficulty for travel. The total of 25 disability studies were categorized three domains: (a) 16 of them related to changing healthcare professionals' attitudes toward PWD through interventions, trainings and curricula inclusion, (b) 5 of them aimed at interventions of improving interactions between patients with disabilities and physicians, and (c) 4 of them about accessing care for PWD to reduce health care barriers via telehealth provided by occupational therapists, psychologists, and counselors. The previous disability studies demonstrated effective interventions aimed at cultural competence and telehealth for PWD respectively rather integrating. However, few empirical studies have explicitly pinpointed needs, interventions, and outcomes of PWD whether multicultural competent healthcare professionals deliver services through inclusive telehealth. Undoubtedly, Butler., et al. reported the similar finding supported by Hilty., et al.'s study [28,29]. Due to the raising attentions and demands of telehealth for PWD during the COVID-19 pandemic, it is a perfect time to facilitate insights and discuss implications about culturally competent and inclusive healthcare access for PWD.

Multiculturally competent healthcare professionals and inclusive telehealth

While developing a disability-inclusive telehealth system, healthcare professionals' multicultural competencies and inclusive technology accommodations and telehealth platform design need to be considered for PWD. The first part is that multicultural competencies of health service providers (e.g. physicians, nurses, therapists, psychiatrists, psychologists, counselors, social workers, health administrators and clinical practitioners) to have awareness, knowledge, skills, and applications working with people with disabilities and chronic disorders, and elders during telehealth provision [30]. Awareness means recognizing the service provider's own culture and bias, which influences the perceptions toward client/customer with different races and ethnicities. Knowledge includes information and understanding of other people's cultures, characteristics, and worldviews. Skills encompass appropriate intervention strategies of interacting with people with diverse characteristics, including building rapport, validation, and advocacy $[27,30]$. Finally, healthcare professionals must apply multicultural competencies while interacting with underrepresented patients/ clients in telehealth services [27]. The application of multicultural awareness, knowledge, and skills would enhance the service quality and positive health care outcomes for patients/clients. Multicultural Competencies of healthcare providers during telehealth play an essential role in facilitating engagement and quality assurance for PWD.

The following strategies should be included in multicultural competencies application while interacting or serving underrepresented population: (a) examine the cultural value, cultural norm, worldview, and bias of one's own and other people regularly, (b) understand and encourage personal identities acceptance and commitment, (c) show respect on people's culture values, (d) develop a good interpersonal relationship with open, patient, understandable, and practical communication to build up rapport and facilitate information exchange, (e) recognize and understand needs, expectations and goals of clients, (f) empathize and validate the conflicts and mistreatment produced by diverse cultures, (g) be transparent and comprehensive to analyze and assist patients with disabilities to make health care decision, (h) provide assistive technology and accommodations for unique needs in telehealth, (i) advocate for underrepresented group about unequal information exchange and resources distribution and (j) expand more contact experiences with diverse population. The ultimate goal of cultural competence application can substantially impact quality of life and well-being of PWD.

Multicultural competence training is substantial for healthcare professionals in their undergraduate or graduate education programs [31]. The multicultural competence training in clinical workplace-based is vital to building up rapport, trust and satisfaction of underrepresented groups. Video conference with adequate etiquette interactions and other friendly Telehealth features for PWD and other underrepresented patients can enhance working alliance between clinical practitioners and patients and bring positive outcomes and satisfaction of vulnerable populations through the devastating COVID-19 pandemic.

The second part is the collaboration between healthcare professionals and telehealth vendors to design disability-inclusive layouts, features, and platforms for underserved populations. For instance, during COVID-19, lack of interpreters' assistance, people with deaf would be difficult to obtain appropriate and accessible health information. The following accommodations by utilizing adequate software or mobile applications can be considered in telehealth delivery for PWD [32]:

- $\quad$ Closed captioning, living captioning or interpreting during synchronous virtual conference for people with deaf. 
- $\quad$ Color accommodation, word magnifier or screen reader features for people with visual impairments and low visions. Reading functions for visual impairments and low visions.

- Information and instructions by visual prompts (images, picture, graphs, animations) with plain language for people with intellectual and cognitive disabilities.

- $\quad$ Structurally and simple instructions for people with autism spectrum disorders.

- Descriptions with visual prompts with few words for people with attention deficit hyperactivity disorder (ADHD) or dyslexia.

Culturally inclusive design on telehealth platforms in health education, health management, and service delivery improves telehealth access and quality assurance for people with disabilities.

\section{Conclusion}

Global pandemic needs everyone's support and effort to survive. It is believed that no one's life can be expendable and disposable. The lives and rights of PWD should be emphasized continuously and advocated in this pandemic. Disability-inclusive policies and integrated services can be constructed for PWD to ensure equity and inclusion according to various types of disabilities, even for underrepresented PWD (e.g. PWD of color, PWD belong to LGBTQIA+ community, PWD living in rural area, and PWD with low SES and poverty). During this COVID-19 pandemic, quality of life, health disparities, morbidity, mortality, the psychological distress of PWD should be examined and call for actions [33]. Multicultural competencies of healthcare providers (e.g. physician, surgeon, psychiatrists, therapists, nurses, counselors) can facilitate a highlyquality working alliance between service providers and patients/ clients. Applying multicultural competences in disability-inclusive telehealth delivery might be an excellence solution to decrease health care disparities for PWD. For educators in the academic or training programs, designing diverse and inclusion curriculum becomes critical to nurture multicultural competencies of healthcare professionals and implement culturally responsive practices. The local states and the US federal governments should stand on the same side to assist healthcare providers in developing disability responsive strategies through the entire pandemic and avoid discrimination and inequality of health care rationing for PWD. Given that telehealth has been increasing potential market and demand, few studies reported utilization perceptions, satisfactions, and effectiveness related to PWD [26]. Further research can explore the telehealth process and outcomes associated with multiculturally competent healthcare professionals, also, comprehensively assess strengths, weakness, opportunities and plans of telehealth services for PWD.

\section{Conflicts of Interest}

The author declares no conflict of interest.

\section{Bibliography}

1. World Health Organization. "World report on disability 2011". World Health Organization (2011).

2. World Health Organization. "Disability considerations during the COVID-19 outbreak". World Health Organization (2020).

3. Brigo, F., et al. "Telemedicine and the challenge of epilepsy management at the time of COVID-19 pandemic". Epilepsy and Behavior 110 (2020): 107164.

4. Erickson W., et al. "2018 Disability Status Report: United States". Ithaca, NY: Cornell University Yang-Tan Institute on Employment and Disability (YTI) (2020): 1-64.

5. Bright T and Kuper HA. "Systematic review of access to general healthcare services for people with disabilities in low and middle income countries". International Journal of Environmental Research and Public Health 15.9 (2018):1879.

6. Krahn GL., et al. "Persons with disabilities as an unrecognized health disparity population". American Journal of Public Health 105.S2 (2015): S198-206.

7. Sakellariou D., et al. "Disability inclusiveness of government responses to COVID-19 in South America: a framework analysis study". International Journal for Equity in Health 19.1 (2020): 1-10.

8. United Nations. "Convention on the rights of persons with disabilities". United Nations (2016).

9. Devandas C. "COVID-19: Who is protecting the people with disabilities? - UN rights expert". United Nations Office of the High Commissioner for Human Rights (2020).

10. National center for immunization and respiratory diseases. "Information for health departments on reporting cases of COVID-19”. Centers for Disease Control and Prevention (2020).

11. Andrews, EE., et al. "No body is expendable: Medical rationing and disability justice during the COVID-19 pandemic". American Psychologist (2020): 1-11.

12. United Nations. "Policy brief: a disability-inclusive response to COVID-19" (2020): 1-18. 
13. O'Connell CM., et al. "Now, more than ever, our community is needed: spinal cord injury care during a global pandemic". Spinal Cord Series and Cases 6.18 (2020): 1-2.

14. Moscoso-Porras MG and German FA. "Association between perceived discrimination and healthcare-seeking behavior in people with a disability". Disability and Health Journal 11.1 (2018): 93-98.

15. Iezzoni LI., et al. "Rural residents with disabilities confront substantial barriers to obtaining primary care". Health Services Research 41.4 Pt 1 (2006): 1258-1275.

16. Ali A., et al. "Discrimination and other barriers to accessing health care: perspectives of patients with mild and moderate intellectual disability and their carers". PLoS One 8.8 (2013): 1-13.

17. Michalopoulou G., et al. "Physicians' cultural competency as perceived by African American patients". Journal of the National Medical Association 101.9 (2009): 893-899.

18. Michalopoulou G., et al. "Linking cultural competence to functional life outcomes in mental health care settings". Journal of the National Medical Association 106.1 (2014): 42-49.

19. Annlee B. "Health care providers' knowledge, attitudes, and self-efficacy for working with patients with spinal cord injury who have diverse sexual orientations". Physical Therapy 88.2 (2008): 191-198.

20. Bashshur RL., et al. "The empirical foundations of telemedicine interventions for chronic disease management". Telemedicine Journal and e-Health 20.9 (2014): 769-800.

21. Aguilera A. "Digital technology and mental health interventions: Opportunities and challenges”. Arbor 191.771 (2015): A210.

22. Hilty DM., et al. "A comparison of in-person, synchronous and asynchronous telepsychiatry: Skills/Competencies, teamwork, and administrative workflow". Journal of Technology in Behavioral Science 5 (2020): 273-288.

23. Agha Z., et al. "Cost effectiveness of telemedicine for the delivery of outpatient pulmonary care to a rural population". Telemedicine Journal and e-Health 8.3 (2002): 281-291.

24. Khubchandani A and Thew D. "Achieving equity in the health of women with disabilities through telehealth: Challenges and benefits". Eliminating Inequities for Women with Disabilities: An Agenda for Health and Wellness. Washington: American Psychological Association (2016): 83-91.

25. Mekonnen WT. "A framework for evaluating telemedicinebased healthcare inequality reduction in Ethiopia: a grounded theory approach". University of South Africa, PhD dissertation (2019).
26. Annaswamy TM., et al. "Telemedicine barriers and challenges for persons with disabilities: Covid-19 and beyond". Disability and Health Journal (2020): 1-3.

27. Wilson KB., et al. "Facilitating application of the multicultural counseling competencies". Rehabilitation Research, Policy, and Education 33.4 (2019): 234-244.

28. Hilty DM., et al. "Telehealth for rural diverse populations: Telebehavioral and cultural competencies, clinical outcomes and administrative approaches". Mhealth 6.20 (2020): 1-19.

29. Butler M., et al. "Chapter 2 Disability population". Improving cultural competence to reduce health disparities, Comparative Effectiveness Review No. 170. (Prepared by the Minnesota Evidence-based Practice Center under Contract No. 290-201200016-I), Agency for Healthcare Research and Quality; (March 2016): 10-30.

30. Sue DW., et al. "Multicultural counseling competencies and standards: A call to the profession". Journal of Counseling and Development 70.4 (1992): 477-486.

31. Michael J and Richardson A. "Healthcare for all: the independent inquiry into access to healthcare for people with learning disabilities". Tizard Learning Disability Review 13.4 (2008): 28-34.

32. Banks LM., et al. "Disability-inclusive responses to COVID-19: Lessons learnt from research on social protection in low- and middle-income countries". World Development 137 (2021): 105178.

33. Lund EM and Kara BA. "Raising awareness of disabled lives and health care rationing during the COVID-19 pandemic". Psychological Trauma: Theory, Research, Practice, and Policy 12.S1 (2020): S210-S211.

\section{Assets from publication with us}

- Prompt Acknowledgement after receiving the article

- Thorough Double blinded peer review

- Rapid Publication

- Issue of Publication Certificate

- High visibility of your Published work

Website: www.actascientific.com/

Submit Article: www.actascientific.com/submission.php Email us: editor@actascientific.com

Contact us: +919182824667 